\title{
Criminologie
}

\section{Portrait des activités délinquantes et de l'usage de substances psychoactives chez des jeunes consultant un centre de réadaptation pour personnes alcooliques et toxicomanes}

\author{
Joël Tremblay, Natacha Brunelle et Nadine Blanchette-Martin
}

Volume 40, numéro 1, printemps 2007

Conduites addictives et crimes

URI : https://id.erudit.org/iderudit/016016ar

DOI : https://doi.org/10.7202/016016ar

Aller au sommaire du numéro

Éditeur(s)

Les Presses de l'Université de Montréal

ISSN

0316-0041 (imprimé)

1492-1367 (numérique)

Découvrir la revue

Citer cet article

Tremblay, J., Brunelle, N. \& Blanchette-Martin, N. (2007). Portrait des activités délinquantes et de l'usage de substances psychoactives chez des jeunes consultant un centre de réadaptation pour personnes alcooliques et toxicomanes. Criminologie, 40(1), 79-104. https://doi.org/10.7202/016016ar
Résumé de l'article

Près de 900 jeunes présentant une problématique sévère de toxicomanie ont été évalués lors de leur demande de service en centre spécialisé. Un portrait de leur consommation de substances psychoactives est dressé et comparé en fonction des types d'encadrement judiciaire auquel est soumis l'individu, mais aussi en fonction du sexe. On constate des variations importantes de consommation de drogues entre les groupes (jeunes contrevenants par rapport aux jeunes en situation de protection ou encore, sous aucun encadrement légal) permettant d'appuyer l'hypothèse de liens entre, d'une part, l'intensité et le type de consommation de substances psychoactives et, d'autre part, la gravité des activités délictueuses. On tente d'identifier la portion de délits commis pour des fins de consommation de substances psychoactives et, donc, d'évaluer la proportion de jeunes qui cesseraient probablement les activités délictueuses si on pouvait les aider à cesser cette consommation de drogues. Les différences entre les sexes sont également mises en évidence. 


\section{Portrait des activités délinquantes et de l'usage de substances psychoactives chez des jeunes consultant un centre de réadaptation pour personnes alcooliques et toxicomanes}

Joël Tremblay

Centre de réadaptation Ubald-Villeneuve / Centre de réadaptation en alcoolisme et toxicomanie de Chaudière-Appalaches, Recherche et intervention sur les substances psychoactives - Québec (RISQ) Joel_Tremblay@ssss.gouv.qc.ca

Natacha Brunelle

Professeure, Département de psychoéducation, Université du Québec à Trois-Rivières, Recherche et intervention sur les substances psychoactives - Québec (RISQ),

Centre international de criminologie comparée (CICC)

Natacha.Brunelle@uqtr.ca

Nadine Blanchette-Martin

Centre de réadaptation en alcoolisme et toxicomanie de Chaudière-Appalaches / Centre de réadaptation Ubald-Villeneuve

Nadine.Blanchette-Martin@ssss.gouv.qc.ca

RÉSUMÉ • Près de 900 jeunes présentant une problématique sévère de toxicomanie ont été évalués lors de leur demande de service en centre spécialisé. Un portrait de leur consommation de substances psychoactives est dressé et comparé en fonction des types d'encadrement judiciaire auquel est soumis l'individu, mais aussi en fonction du sexe. On constate des variations importantes de consommation de drogues entre les groupes (jeunes contrevenants par rapport aux jeunes en situation de protection ou encore, sous aucun encadrement légal) permettant d'appuyer l'hypothèse de liens entre, d'une part, l'intensité et le type de consommation de substances psychoactives et, d'autre part, la gravité des activités délictueuses. On tente d'identifier la portion de délits commis pour des fins de consommation de substances psychoactives et, donc, d'évaluer la proportion de jeunes qui cesseraient probablement les activités délictueuses si on pouvait les aider à cesser cette consommation de drogues. Les différences entre les sexes sont également mises en évidence. 
ABSTRACT - Nearly 900 heavy alcohol or drug adolescent users were evaluated at their admission into a substance abuse center. Description of their alcohol and drug use is done in interaction with the legal status in which they are embedded but also by sex. Differences are observed between groups (offenders versus the child welfare or without legal restrictions). Those differences give support to the presumed link between severity of substances abuse and importance of criminality. Efforts are made to calculate proportions of subjects for which criminal activities would presumably reduce or stopped by the instauration of abstinence. Differences between sexes are also analyzed.

De nos jours, il n'est pas rare d'entendre aux nouvelles ou de lire dans les journaux qu'un jeune a commis un acte délictueux sous l'influence de la drogue ou qu'il a commis un vol pour se procurer l'argent nécessaire à l'achat de sa dose. La consommation de psychotropes est souvent montrée du doigt, à tort ou à raison, lorsqu'il est question de délinquance juvénile.

Qu'en est-il au juste de l'ampleur et de la nature de cet usage de psychotropes ainsi que de la délinquance juvénile? Cet article propose essentiellement de fournir une description de la délinquance et de l'usage de substances psychoactives chez des jeunes en traitement pour des problèmes de toxicomanie ainsi que de l'interaction entre ces deux formes de déviance. Des différences en fonction du sexe et du type d'encadrement judiciaire sont également abordées.

\section{Usage de drogues}

L'Enquête sur les toxicomanies au Canada (ETC; Adlaf et al., 2005) conduite en 2004 révèle qu'environ $87 \%$ de la population québécoise âgée de 15 à 19 ans avait déjà consommé de l'alcool (Demers et Poulin, 2005). Presque $55 \%$ des jeunes canadiens de 15 à 19 ans avaient consommé du cannabis au moins une fois dans leur vie (Patton et Adlaf, 2005 ) alors que 20,6\% avaient déjà consommé de la cocaïne, du speed, de l'ecstasy, des hallucinogènes ou de l'héroïne (Adlaf et Lalomiteanu, 2005).

Selon les résultats obtenus dans le cadre de l'étude québécoise de l'Institut de la statistique du Québec, conduite également en 2004, 63 \% des élèves des écoles secondaires québécoises (12 à 17 ans) avaient consommé de l'alcool l'année précédente. Pour ce qui est des autres drogues (incluant le cannabis), cette proportion était de 36\% (Pica, 2005). Le cannabis était de loin la drogue illégale la plus consommée 
au cours des douze derniers mois (36\%). Environ $5 \%$ des élèves québécois présentaient une consommation problématique alors que $10 \%$ dévoilaient une consommation à risque de le devenir (Pica, 2005).

Les proportions de filles et de garçons consommateurs québécois sont très semblables (Guyon et Desjardins, 2005). Les drogues de prédilection de chacun diffèrent toutefois un peu. En termes de fréquence et d'intensité de l'usage d'alcool et d'autres drogues, les garçons rapportent boire plus souvent et se saouler également plus souvent (Guyon et Desjardins, 2005). Ils sont aussi proportionnellement plus nombreux à consommer du cannabis quotidiennement (Guyon et Desjardins, 2005). L'enquête de 2004 de l'Institut de la statistique du Québec (Pica, 2005) révèle que les garçons sont plus nombreux que les filles à développer des problèmes d'usage d'alcool et d'autres drogues.

Quoique les taux de prévalence de la consommation de substances psychoactives chez les élèves québécois ne soient pas négligeables, ils sont de beaucoup inférieurs à ceux obtenus avec des sous-groupes de jeunes judiciarisés au Québec (Pauzé et al., 2000; Cousineau et al., 2005) et aux États-Unis (Loeber et al., 1998). Ainsi, parmi les jeunes nouvellement pris en charge par les centres jeunesse du Québec, le taux de consommation de drogues illicites à vie s'élève à $78 \%$ (Pauzé et al., 2000). Les drogues illicites les plus consommées par les jeunes contrevenants sont, dans l'ordre: le cannabis, les hallucinogènes, les amphétamines et la cocaïne (Cousineau et al., 2005; Laventure et al., 2006).

Il est toutefois difficile d'obtenir des informations plus précises concernant les consommateurs davantage problématiques, telles que les taux de prévalence des diagnostics d'abus ou de dépendance ${ }^{1}$. Certaines études rapportent des taux élevés de jeunes aux prises avec une consommation problématique de substances psychoactives (SPA). Ainsi, certains éducateurs en centre jeunesse estiment que $80 \%$ des jeunes auprès de qui ils travaillent présentent une consommation problématique de psychotropes (Brochu, 2006). Selon Laventure, Déry et Pauzé (2006), $25 \%$ des adolescents desservis par les centres jeunesse du Québec présentent une consommation problématique de psychotropes. Les travaux de Laventure et Pauzé (2003) révèlent que, comparativement

1. Dans cet article, l'usage problématique d'alcool et d'autres drogues est notamment caractérisé par l'abus ou la dépendance à ces substances, en fonction des critères du DSMIV-TR. 
aux jeunes qui y sont dirigés selon la Loi de la protection de la jeunesse, ceux qui y sont adressés selon la Loi sur les jeunes contrevenants (LJC) sont plus nombreux à avoir consommé de l'alcool et du cannabis au cours de leur vie, sont plus nombreux à consommer de l'alcool de façon hebdomadaire, sont plus nombreux à présenter une consommation à risque de devenir problématique et présentent un profil d'usage de drogues autres que l'alcool plus détérioré ${ }^{2}$.

\section{Délinquance}

Des auteurs comme Fréchette et Leblanc (1987) avaient déjà montré que la très grande majorité des adolescents commettent à un moment ou un autre au moins un acte délictueux: qu'il s'agisse de voler un paquet de gomme au dépanneur, de faire des graffitis dans un parc, de se battre à l'école, de voler un vélo ou de faire une introduction par effraction. Une définition légale de la délinquance est adoptée dans cet article. La délinquance réfère à l'adoption de comportements pour lesquels une sanction pénale est prévue par une ou des lois (Picca, 1993). En ce qui concerne l'adolescence, il aurait été aisé d'assimiler le terme délinquance à celui du trouble des conduites, la documentation à ce sujet étant abondante, mais ce dernier réfère à une multitude de comportements qui ne sont pas nécessairement punissables par des lois, tels que ne pas respecter le couvre-feu imposé par les parents ou faire l'école buissonnière (DSM-IV-TR). De plus, la délinquance ne peut pas être définie seulement en termes psychiatriques, la sociologie, la criminologie et la psychoéducation ayant toutes trois contribué significativement et de façon complémentaire à la compréhension de ce phénomène.

Même si les infractions contre la propriété (vols, fraudes, méfaits, etc.) sont celles pour lesquelles les adolescents canadiens sont de loin le plus arrêtés (35\% en 2003-2004 selon Statistique Canada, 2005), une augmentation considérable des arrestations de mineurs pour des délits de violence a été enregistrée de 1988 à 1998 (Savoie, 1999) et se situait à 19\% en 2003-2004 (Statistique Canada, 2005). Il demeure que, globalement, la prévalence de la criminalité est en baisse et les infractions contre les biens commises par les jeunes contrevenants ont

2. Ils obtiennent un score composé à l'IGT-Ado significativement plus élevé pour l'échelle d'usage de drogues. 
affiché de très importantes baisses dans les années 1990 (Pottie et al., 2005). Entre 1991 et 2003, le nombre total de causes traitées devant le Tribunal de la jeunesse a chuté de $33 \%$ (Thomas, 2005) 3 .

Les filles s'impliquent davantage dans des délits mineurs tels que le vol à l'étalage et sont plus nombreuses que leurs confrères à se prostituer. Malgré une augmentation des délits de violence notée chez les filles ces dernières années, une plus grande proportion de garçons que de filles commettraient des voies de fait, par exemple (Savoie, 1999). Ces dernières abandonnent plus rapidement leur trajectoire délinquante que les garçons (Lanctôt et Leblanc, 2000).

La délinquance et la judiciarisation semblent plus communes chez les jeunes en traitement pour leur toxicomanie que dans la population générale (Brochu, 2006). Une étude québécoise menée auprès de jeunes québécois dans un centre public de réadaptation pour alcoolisme et autres toxicomanies révèle que $45 \%$ des filles et $54 \%$ des garçons de la clientèle disent avoir déjà été inculpés pour des délits qu'ils ont commis (Bertrand et al., 2004).

\section{Associations drogues et crimes}

D'abord, si on s'attarde aux caractéristiques des individus présentant la double problématique drogue-crime ${ }^{4}$, on constate notamment qu'ils ont adopté au moins une de ces conduites déviantes à un âge très précoce (Hawkins et al., 1992). Les jeunes qui s'impliquent à la fois dans la délinquance et la consommation de drogues illicites ont généralement commencé par la commission de délits mineurs, l'usage de drogues illicites apparaissant un peu plus tard dans leur trajectoire, l'inverse étant aussi possible (Brochu, 2006). Ils présentent également des troubles de santé mentale ainsi qu'une résistance plus marquée au traitement (Germain et al., 1999; Pratt et al., 2003).

La délinquance et la consommation problématique d'alcool ou d'autres drogues partagent plusieurs facteurs de risque communs, ce qui peut expliquer leur taux de concomitance très élevé (Born et Gavray, 2002). Parmi les facteurs de risque communs bien documentés, on note

3. Il ne s'agit pas ici de prétendre que cette chute de la judiciarisation reflète nécessairement une baisse de la délinquance juvénile. Elle peut plutôt refléter des changements dans les politiques, les lois et les pratiques policières et judiciaires à l'égard des jeunes.

4. La documentation plus générale sur le trouble des conduites à l'adolescence est ici également pertinente. 
une relation conflictuelle parent/enfant, la précocité des expériences déviantes, l'association à des pairs déviants, une estime de soi négative et des problèmes scolaires (Hawkins et al. 1992; Vitaro et al., 2000). Ce courant psychosocial sur les facteurs de risque est venu soutenir l'affirmation selon laquelle l'usage de drogues est un facteur de risque de la délinquance et vice-versa. L'usage de drogues peut augmenter les probabilités qu'une personne s'engage dans une carrière délinquante persistante et grave (Brochu, 2006). Certains auteurs soutiennent un modèle causal à cause commune selon lequel la personnalité antisociale ou encore l'association à des pairs déviants sont des facteurs de risque communs à la délinquance et à l'usage de drogues (Brochu, 2006). Comme la personnalité antisociale est un diagnostic psychiatrique applicable aux adultes, notons que le pendant à l'adolescence est le trouble des conduites. Ce dernier est le trouble de santé mentale du DSM-IV-TR le plus étroitement associé à l'usage de psychotropes à l'adolescence (Laventure, 2006).

Par ailleurs, il semble que certaines personnes peuvent abandonner un comportement sans cesser complètement l'autre (Brochu, 2006). C'est ainsi que des ex-toxicomanes continuent de commettre des délits lucratifs puisqu'ils sont plus payants, plus faciles ou encore, le font par habitude. Des ex-délinquants peuvent ainsi continuer de consommer des drogues illicites, car ils en retirent encore suffisamment de plaisir et peuvent maintenant financer cette habitude par des sources légales de revenus. Lanctôt (2005) a bien documenté que certaines filles délinquantes abandonnent assez rapidement leurs activités illégales pour passer à une autre forme de déviance dominante telle que l'usage abusif d'alcool et d'autres drogues, ou encore la grossesse précoce.

En général, les corrélations entre usage de drogues et délinquance sont directement proportionnelles, montrant que plus un jeune consomme, plus il s'implique dans la délinquance. La nature et l'explication des liens drogue-crime ne sont pas statiques, elles évoluent au fil de l'adolescence et de l'âge adulte. Elles dépendent notamment de la fréquence et de la gravité de la consommation. Le modèle intégratif de Brochu (2006) tient compte justement de cette évolution à travers, entre autres, des stades de progression des liens drogue-crime: occurrence (consommation occasionnelle), renforcement mutuel (consommation régulière) et économico-compulsif (abus et dépendance de substances psychoactives). Le modèle drogue-crime élaboré par Brunelle et ses collaborateurs (2005) concerne plus spécifiquement les adolescents et 
suit sensiblement la même logique selon laquelle plus un jeune consomme et abuse des drogues, plus la nature des liens drogue-crime qu'il décrit évolue. Ces deux modèles illustrent notamment que c'est généralement dans les cas de dépendance à des drogues coûteuses, sans revenu légal suffisant pour la financer, qu'on peut établir un lien de causalité plus direct selon lequel la délinquance est «causée» par l'usage de drogues des individus.

Selon Brunelle et ses collaborateurs (2000), trois types de relations drogue-crime émergent du récit de vie de jeunes québécois rencontrés en centre jeunesse, en centre de traitement de la toxicomanie et en maison de jeunes. D'abord, certains jeunes affirment l'absence d'une relation drogue-crime dans leur trajectoire (modèle corrélationnel sans cause commune) ou encore décrivent une relation qui passe par l'association à des pairs déviants (modèle corrélationnel avec cause commune décrit plus haut). C'est ainsi que des jeunes expliquent qu'ils consomment et commettent des délits, car les membres de leur groupe de pairs adoptent ces deux types de comportements déviants. Ces derniers types de relations drogue-crime pourraient supporter l'idée selon laquelle drogue et délinquance constituent deux manifestations d'un même style de vie déviant (Cormier, 1993; Brochu et Brunelle, 1997). Aussi, une relation psychopharmacologique surtout utilitaire constitue un cas de figure: des jeunes disent consommer pour faciliter la commission d'un délit (se donner du courage, se déculpabiliser, oublier) ou pour avoir plus de plaisir en le commettant. En ce sens, Guyon et Desjardins (2002) mentionnent que $37 \%$ des élèves québécois présentant une consommation à risque ou déjà problématique $e^{5}$ ont commis un geste délinquant alors qu'ils étaient sous l'influence d'une ou des drogues. Enfin, une autre possibilité est une relation drogue-crime monétaire. Mentionnons que chez certains jeunes, ce serait les revenus issus de leur délinquance lucrative qui les auraient poussés initialement à expérimenter l'usage de drogues. Par ailleurs, la plupart des jeunes consommateurs de l'étude de Brunelle et ses collègues (2000), particulièrement ceux ayant développé des problèmes de toxicomanie, affirment qu'il leur est arrivé de s'adonner à une délinquance lucrative afin de payer leur drogue. Le populaire modèle drogue-crime économico-compulsif de Goldstein (1985), repris par Brochu (2006), est ici évoqué. En ce sens, 
l'étude états-unienne DATOS-A révèle que $53 \%$ des jeunes toxicomanes ont commis des crimes pour acheter des drogues dans l'année précédant leur traitement (Farabee et al., 2001). La vente de drogues constitue un des délits lucratifs les plus communs chez des jeunes en centre de traitement pour la toxicomanie (Kinlock et al., 2004).

\section{Hypothèses}

Sur la base du lien observé entre délinquance et abus de substances, il est postulé que, parmi les jeunes en centre spécialisé de traitement pour toxicomanes, une plus grande ampleur de problèmes de consommation d'alcool et d'autres drogues (quantité, fréquence, conséquences négatives, etc.) sera présente au sein du sous-groupe de jeunes présentant un profil de comportements délictueux (i.e., suivi en centre jeunesse sous la Loi sur les jeunes contrevenants ${ }^{6}$ ) que chez les autres. Compte tenu des observations étayant les différences entre les garçons et les filles quant à l'abus d'alcool et d'autres drogues, des comparaisons liées au sexe seront menées quant à la sévérité du profil de consommation d'alcool et d'autres drogues. Nous voulons également évaluer empiriquement le bien-fondé d'un des résultats obtenus par Brunelle et ses collaborateurs (2000) selon lequel la plupart des jeunes consommateurs s'adonnent à une délinquance de type lucratif notamment pour payer l'achat de drogues?.

\section{Méthodologie}

\section{Procédures}

Les données analysées dans le cadre de cette étude sont des données rétrospectives portant sur l'évaluation de 890 jeunes inscrits consécutivement de mars 1999 à mars 2003 au sein des services spécialisés en toxicomanie de la région de Québec ${ }^{8}$. Tous ces jeunes ont préalablement

6. Au moment de la cueillette des informations, la loi en vigueur était la Loi sur les jeunes contrevenants (LJC), qui est maintenant devenue la Loi sur le système de justice pénal pour les adolescents (LSJPA). Tout au long de l'article, l'acronyme LJC sera toutefois utilisé pour rendre compte du contexte de cette étude.

7. Ce n'est pas le seul type de liens drogue-crime observé par les auteurs, mais c'est celui sur lequel nous pouvons travailler à partir des données de cette étude.

8. Les données concernant 91 évaluations n'ont pas été retenues compte tenu de l'absence d'information importante ou la présence d'annotations contradictoires au sujet de leur statut quant à la LPJ ou la LJC. 
été dépistés à l'aide du questionnaire DEP-ADO (Landry et al., 2004) dans divers milieux (scolaire, centre jeunesse de Québec, CLSC), indiquant ainsi que leur consommation de substances psychoactives a atteint un niveau pour lequel des services spécialisés en toxicomanie semblent nécessaires. De plus, le jeune a accepté d'être évalué en vue de recevoir ces services spécialisés. Une fois le dépistage fait, un évaluateur de l'équipe régionale d'évaluation des jeunes toxicomanes (Programme accès jeunesse toxicomanie [PAJT]) rencontre l'adolescent. Cette équipe est formée de cliniciens de tous les centres de la région de Québec offrant des services spécialisés en toxicomanie aux jeunes, soit le Centre de réadaptation Ubald-Villeuneuve, le Centre JeanLapointe pour adolescents de Québec $^{9}$ et Portage-Jeunes-Québec. Le Centre jeunesse de Québec ne procède pas à l'évaluation des participants, mais joue un rôle important quant au suivi de ce programme. L'évaluateur fixe une rencontre au jeune en vue d'évaluer l'ampleur de sa consommation de SPA et, par la suite, de l'orienter vers une des ressources mentionnées en fonction de critères préalablement fixés. L'évaluation et l'orientation sont finalement révisées par la coordonnatrice de l'équipe afin de s'assurer de la qualité des données recueillies et de la justesse de l'orientation. Les informations issues de l'évaluation sont, par la suite, saisies dans les banques de données du CRUV par des assistants de recherche.

L'instrument utilisé est l'Indice de gravité d'une toxicomanie (IGTAdo) (Landry et al., 2000). Les cliniciens membres de l'équipe d'évaluation ont reçu un entraînement rigoureux à l'utilisation de l'IGT-Ado. Deux ou trois membres du groupe d'évaluateurs participent à des rencontres annuelles de mise à jour dans l'utilisation de l'IGT-Ado (auprès de l'équipe de recherche ayant développé l'IGT-Ado, soit le RISQ) et doivent faire part au reste de l'équipe des ajustements faits à l'instrument de mesure.

\section{Instrument de mesure}

L'IGT-Ado (Landry et al., 2000) évalue la qualité du fonctionnement d'un jeune au sein de dix sphères de vie. Chacune de ces sphères est évaluée par des points portant sur la situation du jeune au cours des 
trente derniers jours et d'autres points portant sur sa situation au cours de la vie (n'incluant pas le dernier mois). Pour chacune des sphères de l'IGT-Ado, les questions sont synthétisées par un indice appelé score composé. Cette cote, référant uniquement aux comportements rapportés pour les 30 derniers jours précédant l'évaluation, permet de quantifier le degré des difficultés récemment éprouvées par un jeune dans chacune des sphères. Les sphères évaluées portent sur la consommation d'alcool (25 points dont 4 sont utilisés pour le calcul du score composé, noté 25/4 dans les autres sphères), la consommation de drogues (84/11), l'état de santé physique $(12 / 3)$, la situation scolaire (14/3), la situation de travail (7/3), les loisirs (6/3), l'état psychologique (39/17), la situation interpersonnelle $(17 / 3)$, la situation familiale $(34 / 3)$, la situation sociale et judiciaire (29/4). La cote concernant les 30 derniers jours est parfois non pertinente lorsqu'une majorité de jeunes logent en établissement (en centre de réadaptation, garde fermée ou ouverte, etc.) ou en situation de placement. C'est la situation des jeunes de la présente étude et c'est pourquoi les habitudes de consommation et les difficultés aux différentes sphères doivent être évaluées à partir des questions ne formant pas le score composé mais plutôt portant sur la dernière année ou au cours de la vie. À la fin de l'évaluation, le clinicien doit se prononcer au sujet des substances causant un problème majeur dans la vie du jeune, c'est-à-dire associées à un diagnostic probable d'abus ou de dépendance au DSM-IV-TR.

La validité de l'instrument est bien établie. Malgré le fait qu'aucune analyse factorielle n'a été menée jusqu'à maintenant, on note des corrélations interéchelles oscillant entre 0,03 et 0,52 , indiquant ainsi une certaine parenté conceptuelle entre quelques échelles, mais une indépendance suffisante pour considérer les sphères comme distinctes (Landry et al., 2000). L'IGT-Ado permet de discriminer les jeunes issus du monde scolaire de ceux consultant en centre de réadaptation en toxicomanie sur la base des scores composés alcool, drogues, occupationnel, état psychologique, relations familiales et système social-judiciaire. Les scores composés santé physique et relations interpersonnelles ne permettent pas de discriminer entre les jeunes des deux groupes.

La fidélité de l'instrument est excellente, avec des corrélations test-retest de 0,81 et plus, à l'exception des sphères interpersonnelle $(\mathrm{r}=, 77)$ et occupationnelle $(\mathrm{r}=, 69)$ pour lesquelles les corrélations sont légèrement plus faibles, mais largement acceptables (Landry et al., 2000). La cohérence interne des échelles est très bonne, présentant des 
coefficients alpha de Cronbach de ,83 et plus pour cinq des huit échelles, les trois autres sphères obtenant des indices de cohérence interne très acceptables (drogues, $\alpha=, 70$; relations interpersonnelles, $\alpha=, 78$; système social et judiciaire, $\alpha=, 75)$.

\section{Participants}

Les participants ont été répartis en fonction de leur statut administratif au Centre jeunesse de Québec. Ce statut, bien qu'imparfait, permet une catégorisation à partir d'informations fiables. D'autres informations issues de l'IGT-Ado permettraient d'évaluer la présence d'actes délictueux comme, par exemple, les questions 6,21 et 22 de la sphère judiciaire, qui portent sur la présence d'arrestations au cours de la vie et sur la proportion de celles-ci pour laquelle le jeune a été accusé, puis reconnu coupable. L'IGT-Ado ne permet pas de connaître le délai depuis ces arrestations et, en ce sens, il est apparu plus fiable d'utiliser le statut légal à titre de témoin des actes délinquants actuels. Il n'y a pas non plus de questions appropriées dans l'IGT-Ado permettant de recueillir de l'information sur les actes délictueux non judiciarisés. C'est pourquoi le statut actuel au regard de la LJC a été retenu. D'autre part, la cooccurrence bien connue des situations de négligence-abus et de délinquance nous a conduits à créer une catégorie de jeunes qui font l'objet d'un suivi dans le cadre des deux lois afin de vérifier si la situation de consommation de SPA est différente pour ces jeunes.

Ainsi, un jeune a été classifié (voir tableau 1) comme étant sous la LPJ s'il a déclaré être sous une mesure de placement ordonné ou volontaire au regard de la LPJ (q. 4, sphère judiciaire, IGT-Ado, v3.1). Un jeune a été classifié comme étant sous la LJC s'il a déclaré être en instance d'accusation, en attente de procès ou d'ordonnance (de décision) (q. 23, sphère judiciaire, IGT-Ado, v3.1) ou s'il a déclaré être sous une mesure quelconque au regard de la LJC (détention provisoire, placement, liberté sous condition, etc.) (q. 26, sphère judiciaire, IGT-Ado, v3.1).

Un jeune répondant aux deux catégories précédentes a été classifié comme étant suivi sous les deux lois. À l'inverse, un jeune n'ayant pas de suivi en vertu d'au moins une des deux lois est classifié comme n'ayant pas été vu au centre jeunesse $(\mathrm{n}=334)$. Il faut rappeler que l'ensemble des informations est vérifié par la coordonnatrice du PAJT qui, à travers de nombreux contacts interétablissements, s'assure de la véracité des informations recueillies lors de l'évaluation. 
L'analyse des caractéristiques des jeunes formant les différentes catégories semble en confirmer l'adéquation, à tout le moins pour isoler le profil de délinquance. Les données du tableau 1 indiquent qu'il n'y a que 7 à $13 \%$ des jeunes qui ne consultent pas au centre jeunesse ou qui sont suivis uniquement sous la LPJ et qui ont déjà été reconnus coupables pour un délit quelconque. Par contre, il est probablement plus fréquent que les jeunes faisant l'objet de mesures pour leur délinquance (uniquement sous la LJC) portent une histoire de négligence ou d'abus, voire de prise en charge sous la LPJ (35\% ayant déjà fait l'objet d'une ordonnance de placement sous la LPJ). Le profil de délinquance est donc plus facile à isoler alors que l'histoire de négligence, d'abus et de prise en charge par la LPJ semble plutôt traverser les différentes catégories.

Les participants se divisent donc en quatre sous-groupes quant à leur situation légale actuelle: 1) ceux ne consultant pas au Centre jeunesse de Québec (CJQ) ( $\mathrm{n}=334) ; 2$ ) ceux consultant au CJQ sous le couvert de la LPJ $(\mathrm{n}=243)$; 3) ceux consultant au CJQ sous le couvert de la LJC $(n=180)$, ou encore 4$)$ sous le couvert des deux lois $(n=133)$.

TA B LEA U 1

Statut des participants au regard de la LPJ et de la LJC au moment de l'évaluation initiale

\begin{tabular}{|c|c|c|c|c|}
\hline & $\begin{array}{c}\text { Non- } \\
\operatorname{CJQ}^{1}(\%=n \\
/ 334)\end{array}$ & $\begin{array}{c}\mathrm{CJQ} / \\
\mathrm{LPJ}^{1}(\%=\mathrm{n} \\
/ 243)\end{array}$ & $\begin{array}{c}\text { CJQ } / \text { LPJ- } \\
\operatorname{LJC}^{1}(\%=n / \\
133)\end{array}$ & $\begin{array}{c}\text { CJQ / LJC } 1 \\
(\%=n / \\
180)\end{array}$ \\
\hline \multicolumn{5}{|l|}{ Loi sur la protection de la jeunesse } \\
\hline - Placement volontaire & - & $48 \%(116)$ & $31 \%(41)$ & - \\
\hline - Placement ordonné & - & $52 \%(127)$ & $69 \%(92)$ & - \\
\hline \multicolumn{5}{|l|}{ Autres informations sphère sociale/LPJ } \\
\hline - A déjà été placé en protection LPJ? & $13 \%(45)$ & $90 \%(220)$ & $91 \%(121)$ & $35 \%(63)$ \\
\hline $\begin{array}{l}\text { - A déjà été abusé physiquement } \\
\text { (avec dommages corporels)? }\end{array}$ & $28 \%(93)$ & $43 \%(104)$ & $48 \% /(64)$ & $28 \%(51)$ \\
\hline - A déjà été abusé sexuellement? & $17 \%(57)$ & $16 \%(39)$ & $30 \%(40)$ & $10 \%(18)$ \\
\hline \multicolumn{5}{|l|}{ Loi sur les jeunes contrevenants ${ }^{2}$} \\
\hline $\begin{array}{l}\text { - En instance d'accusation, en attente } \\
\text { de procès ou d'ordonnance }\end{array}$ & - & - & $59 \%(79)$ & $\begin{array}{l}60 \% \\
(108)\end{array}$ \\
\hline $\begin{array}{l}\text { - Détention provisoire, placement en } \\
\text { garde ouverte ou fermée }\end{array}$ & - & - & $52 \%(69)$ & $18 \%(32)$ \\
\hline $\begin{array}{l}\text { - Liberté sous condition, probation, } \\
\text { autres }\end{array}$ & - & - & $22 \%(29)$ & $\begin{array}{l}58 \% \\
(104)\end{array}$ \\
\hline
\end{tabular}




\begin{tabular}{lllll}
\hline Autres informations sphère judiciaire & & & & \\
- A déjà été arrêté ou reçu une & $23 \%(76)$ & $24 \%(58)$ & $82 \%(109)$ & $92 \%$ \\
$\quad$ sommation à comparaître? & & & & $(165)$ \\
- A déjà été accusé pour une de ces & $7 \%(23)$ & $16 \%(38)$ & $81 \%(108)$ & $77 \%$ \\
$\quad \begin{array}{l}\text { arrestations? } \\
\text { - A déjà été reconnu coupable pour }\end{array}$ & $7 \%(22)$ & $13 \%(31)$ & $61 \%(81)$ & $\begin{array}{c}(139) \\
\quad \text { l'une de ces accusations? }\end{array}$ \\
\hline
\end{tabular}

\footnotetext{
${ }^{1}$ Non-CJQ: participants ne faisant l'objet d'aucun suivi, mesure ou prise en charge par le Centre jeunesse de Québec; CJQ/LPJ: participants faisant l'objet d'une prise en charge par le Centre jeunesse de Québec en vertu de la Loi sur la protection de la jeunesse; CJQ/LPJ-LJC: participants faisant l'objet d'une prise en charge par le Centre jeunesse de Québec en vertu de la Loi sur la protection de la jeunesse et de la Loi sur les jeunes contrevenants; CJQ/LJC: participants faisant l'objet d'une prise en charge par le Centre jeunesse de Québec en vertu de la Loi sur les jeunes contrevenants.

${ }^{2}$ Les individus peuvent à la fois être en instance d'accusation, en attente de procès ou d'ordonnance et, en même temps, en détention provisoire, sous une mesure de placement en garde ouverte/fermée, en liberté sous condition, en probation, etc.
}

Il est possible de constater, à la lecture du tableau 1, qu'une plus grande proportion des jeunes CJQ/LPJ-LJC, lorsqu'on les compare à ceux faisant uniquement l'objet de mesures sous la LPJ, sont sous une ordonnance de placement en vertu de la LPJ $(69 \%$ contre $52 \%), \chi^{2}(1)=14,9$; $\mathrm{p}<, 001$. Quant aux mesures sous la LJC, des jeunes étant sous les deux lois (LPJ et LJC), une plus grande proportion d'entre eux (groupe LPJLJC) sont sous des mesures de détention provisoire, placement en garde ouverte ou fermée ( $52 \%$ ) qu'au sein du groupe d'individus uniquement sous la LJC $(18 \%), \chi^{2}(1)=83,8 ; \mathrm{p}<, 0001$.

Les deux tiers des participants sont des garçons $(65,5 \%)$, mais cette distribution varie largement en fonction des sous-groupes de l'échantillon, $\chi^{2}(3)=50,8 ; p<, 0001$. Ainsi, on note une distribution presque équitable en fonction du sexe chez les jeunes n'étant pas sous une mesure du CJQ ( $54 \%$ de garçons) alors que la proportion de garçons est de $63 \%$ chez les jeunes CJQ/LPJ, de $76 \%$ chez les jeunes sous les deux lois et de $83 \%$ chez les jeunes uniquement sous des mesures dans le cadre de la LJC. Même si les groupes se différencient statistiquement quant à la moyenne d'âge (ANOVA, $F(3,886)=40,1 ; \mathrm{p}<, 001)$, on note des écarts très minimes, la moyenne pour tout l'échantillon étant de 15,3 ans (é. t. $=1,3$ ) et les écarts de moyennes entre les sous-groupes ne dépassant pas 0,6 année. Au moment de l'évaluation, la grande majorité des individus ne faisant pas l'objet d'un encadrement par le CJQ vivent à leur domicile (93\%), tout comme les jeunes uniquement sous une mesure de la LJC (76\%), et ce, sous divers arrangements (mère et père, mère seule, père et conjointe, etc.). Par contre, moins de $2 \%$ 
des jeunes sous une mesure de la LPJ (avec ou sans mesure sous la LJC) vivent à leur domicile. La grande majorité demeure plutôt en centre de réadaptation en CJ $(84 \%$ à $86 \%)$, ou dans une ressource telle qu'une famille d'accueil, un centre d'hébergement ou une ressource communautaire $(6 \% \text { à } 10 \%)^{10}$.

\section{Résultats}

Des comparaisons de la fréquence de consommation de différentes SPA (voir tableau 2) sont menées tout en contrôlant pour la covariance attribuable au sexe (critère de Pillai $=0,05 ; F(5,880)=9,54 ; \mathrm{p}<, 0001$; voir tableau 3 pour les comparaisons en fonction du sexe) et à l'âge (critère de Pillai $=0,10 ; F(5,880)=20,19 ; \mathrm{p}<, 0001$ ). Les résultats à la MANCOVA révèlent un effet global lié aux groupes à l'étude (critère de Pillai $=0,05), F(15,2646)=2,96 ; p<, 001$. Les ANOVA permettent de noter des différences entre les groupes quant à la consommation de cannabis: $F(3,884)=5,36 ; \mathrm{p}<, 001$, de cocaïne: $F(3,884)=6,08 ; \mathrm{p}<, 001$ et d'hallucinogènes: $F(3,884)=5,27$; $\mathrm{p}<, 01$. Les analyses de contrastes a posteriori ont été menées de manière à comparer chaque groupe au groupe de jeunes uniquement sous la LJC. Parmi les consommateurs de cannabis, on observe que les deux groupes incluant des jeunes sous la LPJ (avec ou sans mesures sous la LJC) consomment moins fréquemment du cannabis que ceux sous la LJC. Au sujet de la consommation de cocaïne et d'hallucinogènes, les jeunes sous la LJC (avec ou sans mesures sous la LPJ) en consomment plus souvent que les autres.

Afin de donner une signification clinique à ces comparaisons, les données sont reprises, mais cette fois en identifiant les proportions de consommateurs fréquents pour chaque SPA (voir tableau 2). À l'aide du test du Chi carré, on y constate que les jeunes sous la LJC (qu'ils soient en plus sous la LPJ ou non) sont plus nombreux à être des consommateurs fréquents (une fois et plus par semaine) de cocaïne ou d'hallucinogènes. Ils sont aussi plus nombreux que les trois autres groupes à recourir au cannabis trois fois par semaine et plus.

10. Un rapport complet décrivant en détail les résultats des participants à toutes les sphères de 1'IGT-Ado a été produit par l'équipe et est disponible auprès du premier auteur. 
TABLEA U 2

\section{Consommateurs d'alcool ou de drogues au cours de la dernière année: proportion et fréquence comparées en fonction du statut au regard de la LPJ et de la LJC}

\begin{tabular}{|c|c|c|c|c|c|}
\hline & $\begin{array}{l}\text { Non-CJQ } \\
(\%=n / \\
334)\end{array}$ & $\begin{array}{l}\text { CJQ/LPJ } \\
(\%=n / \\
243)\end{array}$ & $\begin{array}{c}\text { CJQ/LPJ-LJC } \\
\left(\begin{array}{c}\%=n / \\
133)\end{array}\right.\end{array}$ & $\begin{array}{l}\text { CJQ/LJC } \\
(\%=n / \\
180)\end{array}$ & $\begin{array}{c}\text { Total } \\
(\%=n / \\
890)\end{array}$ \\
\hline \multicolumn{6}{|l|}{$\begin{array}{l}\text { Fréquence de consommation des } \\
\text { SPA depuis une année } \\
(0=\text { jamais } ; 3=1 \text { à } 2 \text { fois } / \text { sem. } \\
5=\text { quotidienne) moyenne (é.t. })^{1}\end{array}$} \\
\hline - Alcool & $1,84(1,1)$ & $1,62(1,1)$ & $1,99(1,2)$ & $1,75(1,0)$ & $1,79(1,1)$ \\
\hline - Cannabis & $3,90(1,3)$ & $3,69^{\mathrm{a}}(1,3)$ & $3,78^{\mathrm{a}}(1,5)$ & $4,21(1,0)$ & $3,89^{* *}(1,3)$ \\
\hline - Cocaïne & $0,37^{\mathrm{a}}(0,9)$ & $0,30^{\mathrm{a}}(0,8)$ & $0,75(1,3)$ & $0,63(1,3)$ & $0,46^{* \star *}(1,1)$ \\
\hline - Stimulants & $0,32(0,9)$ & $0,26(0,8)$ & $0,35(1,0)$ & $0,38(1,0)$ & $0,32(0,9)$ \\
\hline - Hallucinogènes & $0,93^{\mathrm{a}}(1,1)$ & $0,80^{\mathrm{a}}(0,9)$ & $1,2(1,2)$ & $1,09(1,3)$ & $0,97^{* *}(1,1)$ \\
\hline \multicolumn{6}{|l|}{$\begin{array}{l}\text { Proportion de consommateurs } \\
\text { fréquents }^{2}\end{array}$} \\
\hline - Alcool (3 fois/sem. et plus) & $13,5 \%$ & $8,6 \%$ & $14,3 \%$ & $8,3 \%$ & $11,2 \%$ \\
\hline - Cannabis (3 fois/sem. et plus) & $75,4 \%$ & $66,3 \%$ & $69,9 \%$ & $85,0 \%$ & $74,0 \% * * *$ \\
\hline - Cocaïne (1 fois/sem. et plus) & $6,6 \%$ & $4,9 \%$ & $12,0 \%$ & $11,7 \%$ & $8,0 \% *$ \\
\hline - Stimulants (1 fois/sem. et plus) & $5,7 \%$ & $6,2 \%$ & $7,5 \%$ & $7,2 \%$ & $6,4 \%$ \\
\hline $\begin{array}{l}\text { - Hallucinogènes (1 fois/sem. et } \\
\text { plus) }\end{array}$ & $18,6 \%$ & $13,2 \%$ & $24,1 \%$ & $23,9 \%$ & $19,0 \%$ * \\
\hline \multicolumn{6}{|l|}{$\begin{array}{l}\text { Proportion de jeunes ayant un } \\
\text { problème avec cette substance, } \\
\text { selon le clinicien }{ }^{2,3}\end{array}$} \\
\hline - Alcool & $32,9 \%$ & $23,5 \%$ & $24,8 \%$ & $29,4 \%$ & $28,4 \%{ }^{\S}$ \\
\hline - Cannabis & $89,5 \%$ & $83,5 \%$ & $75,9 \%$ & $88,9 \%$ & $85,7 \% * *$ \\
\hline - Cocaïne & $9,9 \%$ & $7,8 \%$ & $18,8 \%$ & $11,7 \%$ & $11,0 \%$ * \\
\hline - Stimulants & $2,7 \%$ & $6,2 \%$ & $2,3 \%$ & $2,2 \%$ & $3,5 \% \S$ \\
\hline - Hallucinogènes & $21,0 \%$ & $24,7 \%$ & $33,1 \%$ & $28,3 \%$ & $25,3 \%$ * \\
\hline \multicolumn{6}{|l|}{ Autres indicateurs } \\
\hline - Injection des drogues ${ }^{2}$ & $3,6 \%$ & $5,3 \%$ & $10,5 \%$ & $8,9 \%$ & $6,2 \% *$ \\
\hline - Surdose $e^{2}$ & $8,5 \%$ & $9,5 \%$ & $12,8 \%$ & $9,5 \%$ & $9,6 \%$ \\
\hline $\begin{array}{l}\text { - Activités illégales pour obtenir } \\
\text { des drogues }{ }^{2}\end{array}$ & $45,3 \%$ & $46,4 \%$ & $63,5 \%$ & $67,6 \%$ & $52,7 \% * * *$ \\
\hline
\end{tabular}

$\mathrm{p}<, 10$ * $\mathrm{p}<, 05^{* *} \mathrm{p}<, 01{ }^{* * *} \mathrm{p}<, 001$

a Ce score est significativement différent de celui du groupe LPJ $(p<, 05)$ (MANCOVA avec analyses de contrastes a posteriori).

1 Comparaisons des moyennes, MANCOVA avec contrôle pour la variance attribuable au sexe et à l'âge.

2 Comparaisons des pourcentages, Chi carré.

3 Les individus peuvent présenter des problèmes avec plus d'une substance. 
Les cliniciens estiment que près de 9 jeunes sur 10 ont des difficultés significatives avec le cannabis, les jeunes sous le couvert des deux lois étant quelque peu moins nombreux dans cette situation ${ }^{11}$. Par contre, ce sont ces derniers qui sont les plus nombreux à avoir des difficultés significatives avec la cocaïne et les hallucinogènes. Le recours à l'injection comme voie d'absorption des drogues s'observe chez une plus grande proportion de jeunes judiciarisés, cette proportion augmentant encore chez ceux qui sont aussi sous la LPJ. De plus, les deux tiers des jeunes sous la LJC (avec ou sans mesures sous la LPJ) ont recours à des activités illégales pour pouvoir obtenir des drogues ${ }^{12}$.

En ce qui a trait aux différences en fonction du sexe (voir tableau 3), on constate, de façon générale, un profil plus sévère de consommation chez les filles. Des MANCOVA, où on contrôle pour la variance attribuable à l'âge, révèlent des différences entre les garçons et les filles quant à la fréquence de consommation des SPA (critère de Pillai $=0,05$; $F(5,883)=9,04 ; \mathrm{p}<, 0001)$. Les ANOVA indiquent que les filles consomment plus fréquemment de l'alcool $(F(1,887)=17,34$; $\mathrm{p}<, 0001)$, de la cocaïne $(F(1,887)=6,28 ; \mathrm{p}<, 05)$ et des hallucinogènes $(F(1,887)=9,04, \mathrm{p}<, 01)$. Elles consomment cependant moins fréquemment du cannabis que les garçons $(F(1,887)=12,91$; $\mathrm{p}<, 001)$. Toujours afin d'illustrer les mêmes données, mais avec des indicateurs plus significatifs sur le plan clinique, les proportions de consommateurs fréquents sont reprises (comparaisons faites à l'aide du Chi carré). On note essentiellement les mêmes tendances, soit une plus grande proportion de filles que de garçons pour ce qui est de la consommation fréquente de diverses SPA, à l'exception du cannabis, que plus de garçons consomment fréquemment. De façon cohérente, pour presque toutes les substances, une plus grande proportion de filles, selon l'estimation du clinicien, avaient des problèmes significatifs, à l'exception toujours du cannabis. Les garçons ont davantage tendance que les filles à recourir à des activités illégales pour se procurer les drogues recherchées.

11. Les comparaisons suivantes sont faites à l'aide du test du Chi carré, les données étant sous un mode binaire.

12. L'information concernant le recours à des activités illégales pour obtenir des drogues provient de la question $\mathrm{n}^{\circ} 18$ de la sphère alcool-drogues où l'on demande: «Au cours des 12 derniers mois, quelles ont été tes sources de revenus pour l'achat de tes consommations?» Puis un choix de réponses est offert dont, entre autres, «Activités illégales». 
TA B LEA U 3

Consommateurs d'alcool ou de drogues au cours de la dernière année: proportion et fréquence comparées en fonction du sexe

\begin{tabular}{|c|c|c|c|}
\hline & $\begin{array}{c}\text { Garçons } \\
(\%=n / 583)\end{array}$ & $\begin{array}{c}\text { Filles } \\
(\%=n / 307)\end{array}$ & $\begin{array}{c}\text { Total } \\
(\%=n / 890)\end{array}$ \\
\hline \multicolumn{4}{|l|}{$\begin{array}{l}\text { Fréquence de consommation des SPA depuis } \\
\text { une année }(0=\text { jamais; } 3=1 \text { à } 2 \text { fois/sem.; } \\
5=\text { quotidienne) moyenne (é. t. })^{1}\end{array}$} \\
\hline $\begin{array}{l}\text { - Alcool } \\
\text { - Cannabis } \\
\text { - Cocaïne } \\
\text { - Stimulants } \\
\text { - Hallucinogènes }\end{array}$ & $\begin{array}{l}1,69(1,1) \\
4,00(1,1) \\
0,41(1,0) \\
0,28(0,9) \\
0,89(1,1)\end{array}$ & $\begin{array}{l}1,97(1,1) \\
3,68(1,4) \\
0,55(1,1) \\
0,39(0,9) \\
1,10(1,2)\end{array}$ & $\begin{array}{c}1,79 * * *(1,1) \\
3,89 * *(1,3) \\
0,46^{*}(1,1) \\
0,32(0,9) \\
0,97 * *(1,1)\end{array}$ \\
\hline \multicolumn{4}{|l|}{ Proportion de consommateurs fréquents ${ }^{2}$} \\
\hline $\begin{array}{l}\text { - Alcool (3 fois/sem. et plus) } \\
\text { - Cannabis ( } 3 \text { fois/sem. et plus) } \\
\text { - Cocaïne ( } 1 \text { fois/sem. et plus) } \\
\text { - Stimulants ( } 1 \text { fois/sem. et plus) } \\
\text { - Hallucinogènes (1 fois/sem. et plus) }\end{array}$ & $\begin{array}{c}9,1 \% \\
78,2 \% \\
6,9 \% \\
5,1 \% \\
16,0 \%\end{array}$ & $\begin{array}{c}15,3 \% \\
66,1 \% \\
10,1 \% \\
8,8 \% \\
24,8 \%\end{array}$ & $\begin{array}{c}11,2 \% * * \\
74,0 \% * * * \\
8,0 \% \S \\
6,4 \% * \\
19,0 \% * *\end{array}$ \\
\hline \multicolumn{4}{|l|}{$\begin{array}{l}\text { Proportion de jeunes ayant un problème avec } \\
\text { cette substance selon le clinicien }{ }^{2,3}\end{array}$} \\
\hline $\begin{array}{l}\text { - Alcool } \\
\text { - Cannabis } \\
\text { - Cocaïne } \\
\text { - Stimulants } \\
\text { - Hallucinogènes }\end{array}$ & $\begin{array}{c}26,1 \% \\
90,4 \% \\
9,1 \% \\
2,2 \% \\
23,0 \%\end{array}$ & $\begin{array}{c}32,9 \% \\
76,9 \% \\
14,7 \% \\
5,9 \% \\
29,6 \%\end{array}$ & $\begin{array}{c}28,4 \%{ }^{*} \\
85,7 \% * * * \\
11,0 \%{ }^{*} \\
3,5 \%{ }^{* *} \\
25,3 \%{ }^{*}\end{array}$ \\
\hline \multicolumn{4}{|l|}{ Autres indicateurs ${ }^{2}$} \\
\hline $\begin{array}{l}\text { - Injection des drogues } \\
\text { - Surdose } \\
\text { - Activités illégales pour obtenir des } \\
\text { drogues }\end{array}$ & $\begin{array}{c}4,3 \% \\
6,0 \% \\
58,8 \%\end{array}$ & $\begin{array}{l}9,8 \% \\
16,5 \% \\
41,3 \%\end{array}$ & $\begin{array}{c}6,2 \% * * \\
9,6 \% * * * \\
52,7 \% * * *\end{array}$ \\
\hline
\end{tabular}

${ }^{\S} \mathrm{p}<, 10{ }^{*} \mathrm{p}<, 05^{* *} \mathrm{p}<, 01{ }^{* * *} \mathrm{p}<, 001$

${ }^{1}$ Comparaisons des moyennes, MANCOVA avec contrôle pour la variance attribuable à l'âge et ensuite, ANOVA.

${ }^{2}$ Comparaisons des pourcentages, Chi carré.

${ }^{3}$ Les individus peuvent présenter des problèmes avec plus d'une substance.

On observe également que plus du double de filles que de garçons utilisent l'injection comme voie d'administration du produit. Une analyse plus poussée des données, où le type d'encadrement légal est croisé par le sexe, permet d'observer que les filles sous la LJC (avec ou sans LPJ) sont plus nombreuses, en proportion, à recourir à l'injection. Ainsi, lorsqu'on compare les proportions de filles ayant recours à l'injection à travers les quatre types de groupes, on note que 3,9\% des filles non- 
CJQ, 13,3\% de celles sous la LPJ, et approximativement 19\% de celles sous les deux lois (LPJ-LJC) ou sous la LJC uniquement s'injectent (voir figure 1). Ces comportements de consommation sont possiblement associés au fait que plus de filles rapportent avoir déjà eu une surdose, c'est-à-dire une consommation si importante qu'elle a nécessité une intervention médicale.

FIGURE 1

Proportion de garçons et de filles ayant recours à l'injection en fonction de leur statut judiciaire

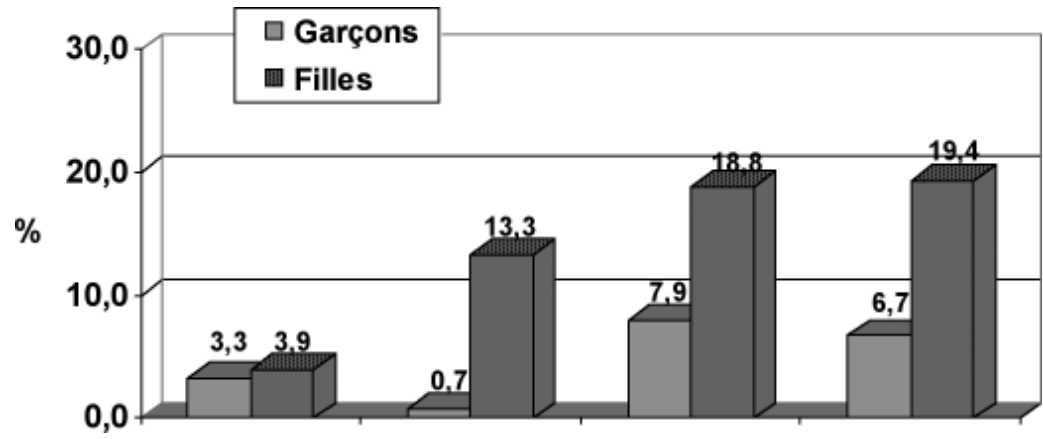

En ce qui a trait aux délits, les jeunes contrevenants (sous la LPJ ou non) sont évidemment plus nombreux à avoir été arrêtés ou à avoir reçu une sommation à comparaître pour différents délits (voir figure 2). On observe des proportions significativement différentes de jeunes ayant été arrêtés ou ayant reçu une sommation à comparaître pour des crimes contre la personne ou violents (infraction sexuelle, voies de fait, négligence criminelle, vol qualifié, viol, homicide) entre certains groupes. En effet, on note que la moitié $(49,6 \%)$ des jeunes sous les deux lois ont commis l'un ou l'autre de ces délits alors que la proportion baisse à $30 \%$ chez les jeunes uniquement sous la LJC, à $9,1 \%$ pour les jeunes sous la LPJ et à 3,9\% chez les jeunes non-CJQ , $\chi^{2}(3)=170 ; \mathrm{p}<, 0001$. Les comparaisons de moyennes au sujet du nombre moyen d'arrestations ou de sommations à comparaître pour ces délits indiquent des différences entre les groupes (en contrôlant pour la variance liée au sexe) (ANOVA, $F(3,885)=27,12 ; \mathrm{p}<, 0001)$. Les comparaisons a posteriori (Tukey) révèlent que les jeunes sous la LJC ont plus d'arrestations de ce type que ceux ne faisant l'objet d'aucune mesure judiciaire. De plus, 
ceux cumulant un encadrement sous la LJC et la LPJ présentent une moyenne significativement plus élevée.

\section{FIG URE 2}

Comparaison, entre les jeunes des quatre groupes, des proportions

d'individus ayant déjà été arrêtés ou ayant reçu une sommation à comparaître pour différents délits

(pourcentages arrondis à l'entier le plus près)

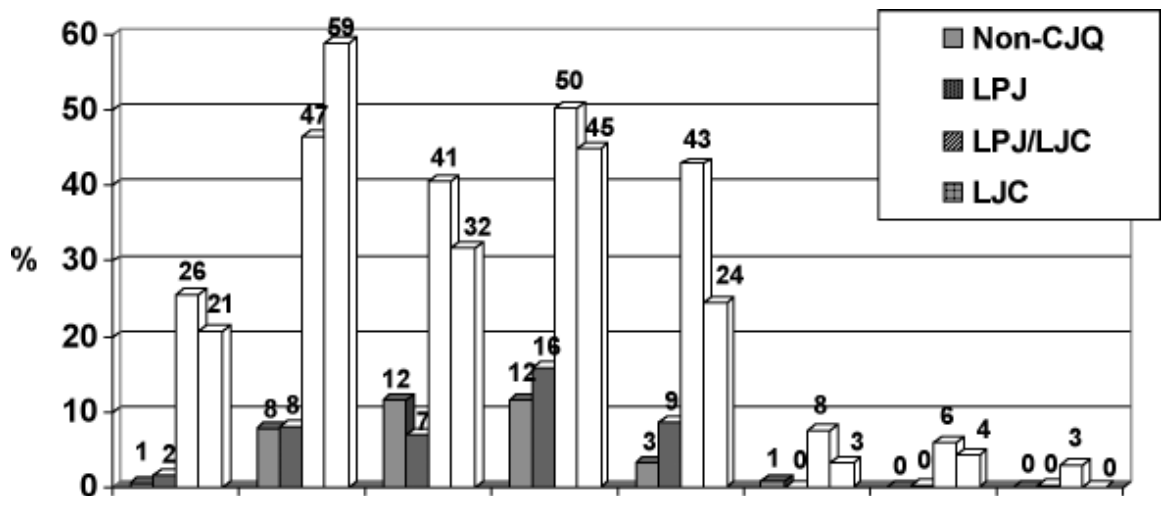

Pour chacun des délits, les distributions sont significativement différentes entre les groupes ( $p<, 001$; Chi carré de Pearson).

Tel que mentionné précédemment, la question $\mathrm{n}^{\circ} 18$ de la sphère alcool-drogues de l'IGT-Ado demande aux jeunes quelles ont été leurs sources de revenus pour se procurer des substances psychoactives et l'un des choix de réponses offert concerne les activités illégales. Ainsi, uniquement pour ceux ayant indiqué avoir recours à des activités illégales pour se procurer les SPA, une tentative est faite afin d'estimer la proportion de jeunes pour qui l'arrêt de consommation d'alcool ou d'autres drogues pourrait être associé à la cessation de commission de délits lucratifs tels que possession-revente de drogues (les données ne permettent pas de répartir les statistiques entre ces deux délits) ou vols (seulement si les jeunes ont rapporté avoir eu recours à des activités illégales pour se procurer des drogues). Les délits directement reliés à la consommation, tels que l'arrestation pour conduite avec facultés affaiblies, sont également considérés à titre de délits directement associés au fait de recourir aux SPA. Ainsi, si on élimine les arrestations 
pour les délits précédemment mentionnés, on obtient une estimation de la proportion d'individus qui commettent des délits non potentiellement associés au besoin de consommation de SPA (i.e. en éliminant les délits lucratifs pour ceux ayant rapporté avoir recours à des activités illégales pour obtenir des SPA). Il est toutefois évident que les conditions de la causalité ne sont pas ici réunies. Cependant, l'estimation permet de noter une baisse des proportions de jeunes qui auraient été arrêtés ou sommés à comparaître, passant de $94 \%$ à $77 \%$ chez les jeunes sous les deux lois, $\chi^{2}(1)=70,4 ; p<0,0001$, mais de façon plus prononcée chez ceux uniquement sous la LJC, passant de $99 \%$ à $63 \%$, $\chi^{2}(1)=2158 ; \mathrm{p}<0,0001$.

\section{Discussion}

Les données actuelles viennent appuyer la thèse selon laquelle la judiciarisation est associée à une gravité plus élevée de la toxicomanie, sans toutefois pouvoir inférer de causalité. Les jeunes sous la LJC (qu'il y ait ou non un encadrement concomitant sous la LPJ) présentent la plus haute proportion de jeunes qui consomment fréquemment du cannabis, de la cocaïne, des hallucinogènes, et un plus grand nombre a recours à l'injection. Le protocole de recherche permet de conclure qu'au sein des jeunes en traitement spécialisé pour abus d'alcool ou autres drogues, la présence de judiciarisation est associée à un abus plus sévère des substances psychoactives. Ainsi, même au sein de ce sous-groupe de jeunes déjà identifiés comme ayant une consommation de SPA importante, les deux phénomènes partagent des taux de concomitance très élevés (Born et Gavray, 2002).

D'autre part, il semble que le cumul des problématiques, dont particulièrement l'ajout de mesures sous la LPJ (et donc d'une problématique de négligence, de violence ou d'abus subis), ne soit pas clairement associé à une aggravation de la situation de consommation de drogues. Ce sous-groupe de jeunes ressemble, sur le plan de l'abus de SPA, beaucoup plus aux jeunes uniquement sous la LJC qu'à ceux sous la LPJ. Cependant, les jeunes de ce groupe (LPJ/LJC) manifestent des différences significatives sur le plan de certains comportements, dont une plus grande propension à avoir commis des crimes violents ou contre la personne. Peu d'études ont porté sur ce sous-groupe particulier de jeunes judiciarisés. Il y aurait avantage à poursuivre les travaux afin de 
mieux comprendre les enjeux liés à un cumul des problématiques associées à la délinquance et à la nécessité d'un encadrement protecteur.

Une quantification des proportions de jeunes chez qui l'association drogues-crimes est uniquement de nature monétaire (i.e. la propension à commettre des délits pour pouvoir acheter des drogues; Brunelle et al., 2000) a été menée. En fait, cette relation semble plus forte chez les jeunes uniquement sous la LJC. Par exclusion, de tous les délits de type lucratif ou associés à la consommation de drogues, près de $40 \%$ des jeunes de ce groupe pourraient hypothétiquement ne plus commettre de délits, si l'abus de substances était traité avec succès. Cette proportion est d'environ $25 \%$ chez les jeunes sous les deux lois (LJC et LPJ). Ces observations permettent aussi de démontrer une délinquance résiduelle importante. Ce résultat confirme, tel que des auteurs comme Brochu (2006) l'ont déjà mentionné, que la consommation de drogues n'est pas toujours responsable de la délinquance des jeunes consommateurs, mais qu'elle explique une portion significative de la délinquance (Kinlock et al., 2004). Toutefois, les données recueillies dans cette étude ne permettent pas de savoir quelles proportions des arrestations des jeunes pour des délits de violence sont attribuables à l'alcool et aux autres drogues, ni de savoir jusqu'à quel point les jeunes étaient intoxiqués au moment de la commission de leurs délits, qu'il s'agisse d'une agression ou d'un vol. Des études portant plus spécifiquement sur ces questions devraient utiliser un questionnaire plus sensible aux types de liens drogue-crime. Les travaux de Brunelle et ses collègues (2000) ont permis de décrire la nature de divers types de relations drogue-crime, mais il manque encore un portrait statistique correspondant, du moins chez les jeunes toxicomanes québécois en traitement. Cousineau et ses collègues (2005) ont fait un tel exercice chez les jeunes en milieu scolaire, mais en se centrant sur les délits de violence uniquement.

Finalement, les comparaisons en fonction du sexe permettent d'observer que les filles, dans l'ensemble, ont un profil de consommation plus important que les garçons. Même si dans la population générale les comparaisons mènent à des conclusions inverses, i.e. une plus grande proportion de garçons aux prises avec un problème de dépendance à l'alcool et autres drogues (Guyon et Desjardins, 2002), il apparaît certain que parmi les jeunes déjà identifiés comme ayant une problématique majeure de consommation, les filles présentent une gravité plus élevée de leur profil. En utilisant l'IGT auprès de clientèles toxicomanes adultes en traitement, des résultats comparables ressortent: les femmes 
toxicomanes arrivent généralement en traitement avec un profil biopsychosocial plus détérioré que les hommes (Guérin, 2000). De plus, des informations soulèvent l'hypothèse que les filles sous la LJC sont particulièrement plus à risque de présenter une toxicomanie plus sévère, et cela, entre autres, par l'observation selon laquelle près d'une fille sur cinq sous la LJC utilise l'injection. Mais cette hypothèse suscitée par nos données devra être vérifiée ultérieurement.

Ces résultats ont différentes implications cliniques. Tout d'abord, la confirmation du lien important qui associe délinquance (ici judiciarisation) et abus de SPA rappelle la nécessité d'avoir une évaluation concomitante de ces deux problématiques, tant auprès des jeunes qui consultent sur la base de leurs actes délictueux qu'auprès de ceux qui font une demande d'aide au sujet de leur consommation de SPA. De plus, la multiplicité des types de liens entre ces deux phénomènes invite à mieux saisir, pour chaque jeune, comment ces deux aspects de sa vie (consommation de SPA et délinquance) sont pour lui associés. En fait, il est nécessaire d'identifier comment chaque dimension influence l'autre, autant que faire se peut, afin d'intervenir sur les bonnes cibles. Finalement, l'intervention dans une sphère de vie (e.g. abus de SPA) devra tenir compte des interactions constantes entre cette sphère et l'autre (e.g. comportements délictueux).

Une limite de l'étude est de concentrer les analyses auprès de jeunes entreprenant un traitement en centre spécialisé pour les toxicomanies. Cette caractéristique du protocole réduit la portée des associations observées entre judiciarisation et intensité de la toxicomanie. En effet, un échantillon de jeunes judiciarisés, mais non orientés vers les centres spécialisés en toxicomanie, aurait permis de mieux évaluer la proportion de jeunes chez qui la judiciarisation (associée ou non à un suivi sous la LPJ) n'est pas, ou est faiblement associée à l'abus de substances. De plus, le protocole transversal utilisé empêche l'évaluation de toute hypothèse de causalité, i.e. une capacité à expliquer comment la délinquance entraîne l'abus de SPA et vice-versa. Un protocole longitudinal permettrait en partie de pallier cette lacune. Une autre limite de l'étude porte sur notre ignorance des activités délictueuses non signalées aux autorités. Il se pourrait que la classification des jeunes en soit changée. Finalement, pour certaines analyses, celles portant sur des données de type binaire, il n'a pas été possible de contrôler la variance attribuable au sexe ou à l'âge. Cependant, les résultats notés dans les deux types d'analyses (comparaison de proportions avec le test du Chi carré ou 
comparaison de scores continus à l'aide d'analyses multivariées avec des covariables) vont exactement dans le même sens.

L'étude permet donc d'appuyer l'hypothèse d'une association plus significative entre délinquance et abus de substances chez les jeunes en traitement pour toxicomanie et sous la LJC que chez les autres. Possiblement que la présence de mesures concomitantes sous la LPJ vient complexifier le portrait clinique, mais il ne semble pas, à la lumière de nos résultats, que cela soit associé à une toxicomanie plus sévère. Les filles ont également un portrait plus grave d'abus de substances que les garçons et certaines données, tout particulièrement celles concernant l'injection, invitent à investiguer plus à fond l'hypothèse d'une interaction entre le genre et le statut légal dans son association à la gravité de la toxicomanie. De futures recherches devraient utiliser un protocole longitudinal pour préciser les liens causaux entre toxicomanie et délinquance. Au sein des futures études, le type d'association drogues-crimes (e.g. économico-compulsif ou pharmaco-psychologique) devrait être évalué chez les jeunes délinquants toxicomanes.

\section{Références}

Adlaf, E. M., Bégin, P., \& Sawka, E. (2005). Enquête sur les toxicomanies au Canada (ETC): une enquête nationale sur la consommation d'alcool et d'autres drogues par les Canadiens: la prévalence de l'usage et les méfaits. Rapport détaillé. Ottawa: Centre canadien contre l'alcoolisme et les toxicomanies.

Adlaf, E. M., \& Lalomiteanu, A. (2005). Other Drug Use and Problems. In Santé Canada et Centre canadien de lutte à la toxicomanie, Enquête sur les toxicomanies au Canada 2004 (55-70).

APA (2000). Diagnostic and Statistical Manual of Mental Disorders: DSM-IV-TR. Washington DC: American Psychiatric Association.

Bertrand, K., Ménard, J.-M., \& Plourde, C. (2004). Synthèse de l'évaluation du programme jeunesse. Recension des écrits et recommandations pour sa révision. TroisRivières: Centre de réadaptation en alcoolisme et toxicomanie Domrémy MCQ.

Born, M., \& Gavray, C. (2002). Drugs and Crime Deviant Pathways. In S. Brochu, C., Da Agra \& M.-M. Cousineau (Eds.), Deviant Trajectories at the Turning Point Between Adolescence and Adulthood (97-114). Hampshire, England: Ashgate.

Brochu, S. (2006). Drogue et criminalité ( $2^{\mathrm{e}}$ édition). Montréal: Les Presses de l'Université de Montréal.

Brochu, S., \& Brunelle, N. (1997). Toxicomanie et délinquance. Une question de style de vie? Psychotropes, 3 (4), 107-125. 
Brunelle, N., Brochu, S., \& Cousineau, M.-M. (2005). Le point sur les trajectoires d'usage de drogues et de délinquance juvénile: des jeunes se racontent. In L. Guyon, S. Brochu \& M. Landry (Éds), Les jeunes et les drogues: usages et dépendances (279-325). Québec: Les Presses de l'Université Laval.

Brunelle, N., Brochu, S., \& Cousineau, M.-M. (2000). Drug-Crime Relation Among Drug Consuming Juvenile Delinquents: A Tripartite Model and More. Contemporary Drug Problems, 27 (4), 835-866.

Cormier, D. (1993). Toxicomanies: Styles de vie. Montréal: Éditions du Méridien.

Cousineau, M.-M., Brochu, S., Fu, S., Houde, V., \& Dufour, C. (2005). Jeunes, drogues et violence: des liens à comprendre. Montréal: Centre international de criminologie comparée.

Demers, A., \& Poulin, C. (2005). Alcohol Use. In, Santé Canada et Centre canadien de lutte à la toxicomanie, Enquête sur les toxicomanies au Canada 2004 (20-32).

Farabee, D., Shen, H., Hser, Y., Grella, C. E., \& Anglin, M. D. (2001). The Effect of Drug Treatment on Criminal Behavior among Adolescents in DATOS-A. Journal of Adolescent Research, 16, 679-696.

Fréchette, M., \& Leblanc, M. (1987). Délinquances et délinquants. Boucherville: Gaëtan Morin Éditeur.

Germain, M., Brochu, S., Bergeron, J., Landry, M., \& Schneeberger, P. (1999). Profils des toxicomanes judiciarisés en traitement dans deux centres de réadaptation publics au Québec. Psychotropes, 7 (1), 71-90.

Goldstein, P. J. (1985). The drugs/violence nexus: A Tripartite Conceptual Framework. Journal of Drug Issues, 14, 493-506.

Guérin, D. (2000). Profil de la clientèle du centre de réadaptation Ubald-Villeneuve. Étude réalisée à partir de l'IGT, 1999. Rapport de recherche non publié. Québec: Centre de réadaptation Ubald-Villeneuve.

Guyon, L., \& Desjardins, L. (2002). La consommation d'alcool et de drogues. In, Institut de la statistique Québec, L'alcool, les drogues, le jeu: les jeunes sontils preneurs? Enquête québécoise sur le tabagisme chez les élèves du secondaire 2000, (Volume 2, 35-64). Québec: Institut de la statistique du Québec.

Guyon, L., \& Desjardins, L. (2005). L'alcool et les drogues chez les élèves québécois de 12 à 18 ans: habitudes, comportements et consommation problématique. In L. Guyon, S. Brochu \& M. Landry (Éds), Les jeunes et les drogues (1-37). Saint-Nicolas: Les Presses de l'Université Laval.

Hawkins, J. D., Catalano, R. F., \& Miller, J. Y. (1992). Risk and Protective Factors for Alcohol and Other Drug Problems in Adolescence and Early Adulthood: Implication for Substance Abuse Prevention. Psychological Bulletin, 112 (1), 64-105.

Kinlock, T. W., Battjes, R. J., \& Gordon, M. S. (2004). Factors Associated with Criminal Severity Among Adolescents Entering Substance Abuse Treatment. Journal of Drug Issues, 34 (2), 293-318.

Lanctôt, N. (2005). Que deviennent les adolescentes judiciarisées près de dix ans après leur sortie du Centre jeunesse? Criminologie, 38 (1), 39-162. 
Lanctôt, N., \& Leblanc, M. (2000). Les trajectoires marginales des adolescents en difficulté: Continuité et changement. Revue internationale de Criminologie et de police technique, 53 (1), 46-68.

Landry, M., Tremblay, J., Guyon, L., Bergeron, J., \& Brunelle, N. (2004). La grille de dépistage de la consommation problématique d'alcool et de drogues chez les adolescents et les adolescentes (DEP-ADO): développement et qualités métriques. Drogues, Santé et Société, 3, 35-67.

Landry, M., Bergeron, J., Provost, G., Germain, M., \& Guyon, L. (2000). Indice de gravité d'une toxicomanie (IGT) pour adolescents et adolescentes. Études des qualités psychométriques. (Rapport $\mathrm{n}^{\circ}$ 23). Montréal, Québec: Recherche et intervention sur les substances psychoactives - Québec.

Laventure, M. (2006). Trouble des conduites et consommation de psychotropes: gravité de la consommation, caractéristiques associées à la cooccurrence des difficultés et persistance du trouble des conduites. Thèse de doctorat au programme de doctorat en éducation de l'Université de Sherbrooke.

Laventure, M., Déry, M., \& Pauzé, R. (2006). Gravité de la consommation de psychotropes des adolescents ayant un trouble des conduites. Criminologie, 39 (2), 165-188.

Laventure, M., \& Pauzé, R. (2003, 24 janvier). La consommation de psychotropes chez les jeunes des centres jeunesse du Québec: Profils de gravité, caractéristiques associées et interventions. Séminaire Jeunesse et Toxicomanie, CIRASST, Montréal.

Loeber, R., Farrington, D. P., Southamer-Loeber, M., Moffitt, T. E., \& Caspi, A. (1998). The Development of Male Offending: Key Findings from the First Decade of the Pittsburgh Youth Study. Studies on Crime and Crime Prevention, 7 (2), 141-171.

Patton, D. \& Adlaf, E. M. (2005). Cannabis Use and Problems. In Santé Canada et Centre canadien de lutte à la toxicomanie, Enquête sur les toxicomanies au Canada 2004 (48-54).

Pauzé, R., Toupin, J., Déry, M., Mercier, H., Cyr, M., Cyr, F., \& Frappier, J.-Y. (2000). Portrait des jeunes inscrits à la charge des Centres jeunesse du Québec et description des services reçus au cours des buit premiers mois (document de travail). Montréal, Québec: Université de Montréal.

Pica, L. (2005). Consommation d'alcool et de drogues. In G. Dubé (Éd), Enquête québécoise sur le tabac, l'alcool, la drogue et le jeu chez les élèves du secondaire, 2004. Quoi de neuf depuis 2002? (95-130). Québec: Institut de la statistique du Québec.

Picca, G. (1993). La criminologie. $3^{\text {e }}$ édition. Paris: Presses universitaires de France.

Pottie Bunge, V., Johnson, H., \& Baldé, T. A. (2005). L'exploration des tendances de la criminalité au Canada. Statistique Canada, 5. Page consultée le 5 mars 2006, <http://www.statcan.ca/francais/research/85-561-MIF>.

Pratt, M., Smith, M., Reigelsperger, R., O'Connor, L. V., Saum, C., Baker, S., $\&$ Reeb, R. N. (2003). Psychosocial treatment alternatives for adolescents with conduct problems. Journal of Psychological Practice, 8, 1-13. 
Savoie, J. (1999). La criminalité de violence chez les jeunes. Juristat, 19 (13). Centre canadien de la statistique juridique, Statistique Canada.

Thomas, J. (2005). Statistiques sur les tribunaux de la jeunesse, 2003-2004. Juristat, 25 (4). Centre canadien de la statistique juridique, Statistique Canada.

Vitaro, F., Carbonneau, R., Gosselin, C., Tremblay, R. E., \& Zoccolillo, M. (2000). L'approche développementale et les problèmes de consommation chez les jeunes: prévalence, facteurs de prédiction, prévention et dépistage. In Brisson, P. (Éd.), L'usage des drogues et la toxicomanie (279-312). Montréal: Éditions Gaëtan Morin. 